\title{
CSF biomarkers differentiate between two forms of frontotemporal lobar degeneration
}

Novel cerebrospinal fluid (CSF) biomarkers can potentially identify patients with one or other of the two main forms of frontotemporal lobar degeneration (FTLD), according to research conducted by John Trojanowski and colleagues at the University of Pennsylvania School of Medicine, USA. Identification of the underlying pathology of FTLD in living patients could "enable early and reliable diagnosis of this disorder, so that therapies, when available, can be given early in the disease process when they are most likely to have the greatest efficacy," says Trojanowski.

Two main forms of FTLD exist: FTLD with TAR DNA-binding protein 43 (FTLD-TDP) pathology and FTLD with tau (FTLD-tau) pathology. To identify CSF biomarkers that discriminate between these two forms of the disease, Trojanowski and colleagues measured the levels of 151 analytes in CSF samples taken from patients with autopsy-confirmed FTLD-TDP or FTLD-tau. Analysis of the results revealed that levels of interleukin (IL)-17, Eotaxin-3, adrenocorticotropic hormone, Fas and agouti-related protein predicted FTLD-TDP pathology. In fact, these five proteins distinguish between FTLD-TDP and FTLD-tau with $82.6 \%$ accuracy, $85.7 \%$ sensitivity and $77.8 \%$ specificity.

\section{4 ...five proteins distinguished between FTLD-TDP and FTLD-tau with $82.6 \%$ accuracy... 77}

Using the above information, the researchers assessed the levels of these analytes in CSF samples from an independent living cohort consisting of 37 patients with behavioral variant frontotemporal dementia (bv-FTD), 22 patients with primary progressive aphasia and 21 patients with corticobasal syndrome. Random forest analysis demonstrated that 53 of these patients were predicted to have FTLD-TDP. Moreover, of the 37 patients diagnosed as having bv-FTD 26 were predicted to have FTLD-TDP pathology. By contrast, among 23 patients with neuropathological information, 12 of 14 patients predicted by their biomarker profile to have FTLD-TDP pathology actually had FTLD-TDP at autopsy, and seven of nine patients predicted to have tau pathology by their CSF profile had an autopsy diagnosis of FTLD-tau.

These results indicate that CSF biomarkers could be used to discriminate between the two main forms of FTLD. Furthermore, "reliable biomarkers will accelerate the pace of drug discovery by making clinical trials more efficient and less costly," concludes Trojanowski.

Nick Jones

Original article Hu, W. T. et al. Novel CSF biomarkers for frontotemporal lobar degeneration. Neurology doi:10.1212/WNL.0b013e318200d78d 